\title{
Impact of the Credentials and Continuing Education of Personnel in the Nuclear Cardiology Laboratory on Its Operational Quality
}

\author{
Saurabh Malhotra, FASNC ${ }^{1}$, Diana M. Sobieraj ${ }^{2}$, April Mann, CNMT, NCT, RT(N) ${ }^{3}$, and Matthew W. Parker ${ }^{4}$ \\ ${ }^{1}$ University at Buffalo, Buffalo, New York; ${ }^{2}$ School of Pharmacy, University of Connecticut, Storrs, Connecticut; ${ }^{3}$ Division of \\ Cardiology, Hartford Hospital, Hartford, Connecticut; and ${ }^{4}$ Division of Cardiovascular Medicine, University of Massachusetts \\ Medical Center, Worcester, Massachusetts
}

Two important factors that have not been systematically evaluated in the delivery of quality imaging services, especially in relation to accreditation, are the specific credentials and continuing medical education/continuing education (CME/CE) of the medical and technical personnel of the nuclear cardiology laboratory. Methods: Facilities performing myocardial perfusion imaging were characterized by setting, size, previous accreditation, and credentials of medical and technical personnel, using data that had been supplied to the Intersocietal Accreditation Commission when these facilities applied for nuclear cardiology accreditation. Multivariable logistic regression was used to compare credentials and CME/CE against the initial accreditation decision (grant or delay). Results: Complete data were available for 1,913 nuclear cardiology laboratories from 2011 to 2014. Laboratories that were granted accreditation had a greater prevalence of Certification Board in Nuclear Cardiology (CBNC)-certified medical directors and specialty-credentialed technical directors. The certification and credentialing of the medical and technical directors, respectively; the CME/CE compliance of the personnel; and the assistance of a consultant with the application were positively associated with the granting of accreditation. Conclusion: A decision to delay accreditation was less likely if the nuclear cardiology laboratory was directed by a CBNC-certified physician and its technologists were credentialed in nuclear cardiology technology or PET. The CME/CE compliance of both the medical and the technical directors was associated with a decision to grant accreditation. The number of years of experience of the medical and technical directors was not associated with a decision to grant accreditation.

Key Words: nuclear cardiology; accreditation; board certification; certified technologists

J Nucl Med Technol 2018; 46:53-58

DOI: 10.2967/jnmt.117.202234

\footnotetext{
Received Sep. 13, 2017; revision accepted Oct. 30, 2017.

For correspondence or reprints contact: Matthew W. Parker, University of Massachusetts Memorial Medical Center, 55 N. Lake Ave., Worcester, MA 01605.

E-mail: matthew.parker@umassmemorial.org

Published online Dec. 22, 2017.

COPYRIGHT (c) 2018 by the Society of Nuclear Medicine and Molecular Imaging.
}

$\mathbf{T}$ he credentials and continuing education of professionals performing nuclear cardiology are key components of quality imaging services $(1,2)$ as emphasized in the Intersocietal Accreditation Commission (IAC) Nuclear/PET accreditation process (3). Specifically, the 2012 IAC Standards (3) required that the medical director be certified by the Certification Board in Nuclear Cardiology (CBNC) (4) or have equivalent training (5) and experience as detailed in the standards. These criteria allow physicians with adequate experience to staff or direct an accredited nuclear cardiology laboratory without being certified by the CBNC. Other medical staff must meet similar criteria. Technical directors may qualify for their position on the basis of state licensure or the certified nuclear medicine technologist (CNMT) or registered technologist (nuclear imaging) (RT[N]) credentials. They may present additional credentials as well, including more specialized training in nuclear imaging (such as the PET or nuclear cardiology technologist [NCT] credentials from the Nuclear Medicine Technology Certification Board, for which the CNMT or registered technologist credentials are a prerequisite (6)) or parallel credentials in related imaging modalities (registered technologist in radiographic imaging, $\mathrm{CT}$, or $\mathrm{MR}[\mathrm{RT}(\mathrm{R}), \mathrm{RT}(\mathrm{CT})$, or $\mathrm{RT}$ (MR), respectively] (7)). The impact of these different credentials on laboratory accreditation and quality has not, to our knowledge, been studied. Nuclear/PET accreditation from the IAC also requires that each physician and technologist submit $15 \mathrm{~h}$ of continuing medical education/continuing education $(\mathrm{CME} / \mathrm{CE})$ credit relevant to the practice of nuclear cardiology in the 3 years before accreditation (3). In one analysis, inadequate $\mathrm{CME} / \mathrm{CE}$ was the most common reason for delayed accreditation (2), being the cause of delay for $30 \%$ of applications. It is less clear whether inadequate $\mathrm{CME} / \mathrm{CE}$ is associated with other deficiencies, such as deficiencies in imaging protocols, the quality improvement processes, or reporting. Using the IAC Nuclear/PET applications database, we investigated nuclear cardiology practice patterns with respect to the credentials of the medical and technical directors and staffs, as well as the CME/CE of both, in relation to peer-reviewed assessments of laboratory quality. 


\section{MATERIALS AND METHODS}

This was a cross-sectional study evaluating data submitted for IAC Nuclear/PET radionuclide myocardial perfusion imaging (MPI) accreditation in the United States from 2011 to 2014. A single accreditation application could be from a nuclear cardiology laboratory at one or multiple sites organized and staffed by a single group of physicians and technologists. IAC Nuclear/PET accreditation is granted for a 3-y period, and facilities must reapply by the same process to maintain accreditation. Among the laboratories applying for accreditation from 2011 to 2014, only those with complete data on the descriptive characteristics of interest, as well as credential and continuing education information for medical and technical personnel, were included for analysis.

According to the IAC Nuclear/PET Standards, medical and technical directors of nuclear cardiology laboratories must meet at least one of the following criteria (3):

- Be board-certified after 1995 (or board-eligible but within 2 y of finishing training) in cardiology and have completed a minimum of a 4-mo formal training program in nuclear cardiology

- Be board-certified in cardiology before 1995 and have training equivalent to level 2 or at least $1 \mathrm{y}$ of nuclear cardiology practice experience, with interpretation of at least 800 studies

- Be certified in nuclear cardiology by the CBNC

- Be board-certified (or board-eligible but within 2 y of finishing training) in nuclear medicine

- Be board-certified (or board-eligible but within 2 y of finishing training) in radiology and have at least 4 mo of nuclear cardiology training

- Be board-certified (or board-eligible but within 2 y of finishing training) in radiology and have special competence in nuclear medicine

- Be board-certified (or board-eligible but within 2 y of finishing training) in radiology and have at least $1 \mathrm{y}$ (full-time equivalent) of nuclear cardiology practice experience, with interpretation of at least 800 studies

- Be board-certified (or board-eligible but within 2 y of finishing training) in radiology and have at least 4 mo of nuclear medicine training, with interpretation of at least 800 nuclear medicine procedures

- Be board-certified (or board-eligible but within 2 y of finishing training) in any other relevant medical specialty and have at least $1 \mathrm{y}$ of nuclear cardiology practice experience, with independent interpretation of at least 800 nuclear cardiology or PET procedures

- If trained before 1995, have 10 y of nuclear cardiology, nuclear medicine, or PET practice experience, with independent interpretation of at least 800 studies within the past $10 \mathrm{y}$

According to the IAC Nuclear/PET Standards, technical directors of nuclear cardiology laboratories must meet one of the following criteria:

- Be a certified nuclear medicine technologist (CNMT, NCT, or PET)

- Be a registered technologist (nuclear) $(\mathrm{RT}[\mathrm{N}])$

- Have a state license to practice as a nuclear medicine technologist if the individual's appointment to technical director took place before January 2010

The specialty of the medical director was categorized as "cardiology" if the individual was certified by the American Board of
Internal Medicine in Cardiovascular Diseases and as "other" if board-certified in radiology or in nuclear medicine or if trained before 1995 and qualified through the experience pathway. To distinguish technical directors who, in addition to being a CNMT or an RT(N), have a specialized credential such as NCT or PET from those who do not, we created categories for them ([CNMT or RT(N)], plus NCT or PET or CNMT or RT(N), only, respectively). To distinguish technical directors with only nuclear medicine-specific credentials from those with a credential in an additional modality (radiology, CT, or MRI), we categorized the latter as multimodality technologists (RT[R], RT[CT], or RT[MR], respectively). All laboratories must have medical and technical directors but do not necessarily have additional staff. If there are other physicians or technologists who participate in nuclear cardiology laboratories but do not have the responsibilities of a director, the IAC Nuclear/PET Standards define the acceptable credentials and CME/CE requirements for them but do not stipulate experience criteria (3).

Descriptive characteristics were compared between those laboratories for which initial accreditation was granted and those for which it was delayed, using the $\chi^{2}$ test for categoric data and the $t$ test or Mann-Whitney test, based on distribution, for continuous data. The primary analysis focused on determining the predictors that accreditation would be granted, whereas the secondary analyses focused on determining, separately, the predictors that deficiencies in reporting and protocols would be found. Multivariate logistic regression models were built to evaluate the association of laboratory characteristics and director credentials and training with the individual dependent variable, with adjustment for the following: number of sites per applicant, annual number of MPI procedures per 1,000 total procedures, number of previous accreditation cycles, whether a consultant assisted with the application, whether the facility was a hospital or a nonhospital, whether the laboratory was also performing general nuclear medicine or PET oncology/neurology, the specialty of the medical director (cardiology vs. other), the number of studies interpreted by the medical director (as quartiles), the CBNC status of the medical director, the $\mathrm{CE}$ compliance of the medical director, the number of medical staff and whether at least $50 \%$ had CBNC certification, the number of CE-compliant medical staff, the technical director credentialing pathway (with or without NCT or PET), the CE compliance of the technical director, the number of technical staff, and the number of technical staff compliant with CE. An exploratory model investigated whether there was an association between the average volume of studies interpreted per physician in each laboratory and a decision to grant accreditation. This variable was substituted for that in the original model - the volume of studies interpreted by the medical director. The results of the multivariate models are presented as adjusted odds ratios with accompanying 95\% confidence intervals. A $P$ value of less than 0.05 was considered statistically significant. Analyses were conducted using SPSS Statistics (version 22; IBM) for Microsoft Windows.

The Institutional Review Board of Hartford Hospital reviewed the investigational plan before any analysis and determined that this study did not qualify as human-subject research.

\section{RESULTS}

In total, 1,913 laboratories were included in the analysis (Table 1). Most (87.9\%) of the IAC Nuclear/PET applicants were nonhospital facilities, and $9.1 \%$ performed general nuclear medicine, oncology, or neurologic procedures in addition 
TABLE 1

Characteristics of Nuclear Cardiology Laboratories Applying for IAC Nuclear/PET Accreditation, 2011-2014

\begin{tabular}{lccc}
\hline \multicolumn{1}{c}{ Characteristic } & $\begin{array}{c}\text { All laboratories } \\
(n=1,913)\end{array}$ & $\begin{array}{c}\text { Accreditation granted } \\
(n=779)\end{array}$ & $\begin{array}{c}\text { Accreditation delayed } \\
(n=1,134)\end{array}$ \\
\hline Facility characteristics & & & \\
Region $(n)$ & & & \\
$\quad$ Northeast & $478(25.0 \%)$ & $203(26.1 \%)$ & $275(24.3 \%)$ \\
Midwest & $329(17.2 \%)$ & $160(20.5 \%)$ & $169(14.9 \%)$ \\
South & $869(45.4 \%)$ & $318(40.8 \%)$ & $551(48.6 \%)$ \\
West & $237(12.4 \%)$ & $98(12.6 \%)$ & $139(12.3 \%)$ \\
Hospital-based laboratory $(n)$ & $232(12.1 \%)$ & $98(12.6 \%)$ & $134(11.8 \%)$ \\
Number of sites (mean) & $1.72(\mathrm{SD}, 3.21)$ & $1.79(\mathrm{SD}, 3.68)$ & $1.68(\mathrm{SD}, 2.84)$ \\
General nuclear medicine or PET $(n)$ & $175(9.1 \%)$ & $60(7.7 \%)$ & $115(14.8 \%)$ \\
MPI volume (mean) & $1,457.58(\mathrm{SD}, 1,638.33)$ & $1,549.5(\mathrm{SD}, 1,769.28)$ & $1,394.5(\mathrm{SD}, 1,539.58)$ \\
Number of application cycles (mean) & $2.82(\mathrm{SD}, 1.01)$ & $2.87(\mathrm{SD}, 1.02)$ & 0.62 \\
Consultant used $(n)$ & $318(16.6 \%)$ & $166(21.3 \%)$ & 0.06 \\
\hline
\end{tabular}

to nuclear cardiology procedures. Most applications $(77.3 \%)$ were for a single-site laboratory, and the largest proportion $(45.4 \%)$ was in the South U.S. census region.

Most medical directors $(69.6 \%)$ were board-certified or board-eligible in cardiovascular diseases by the American Board of Internal Medicine. The remainder were qualified under the experience pathway (with training in cardiology before 1995 and with over $10 \mathrm{y}$ of practice experience [15.0\%]) or were board-certified or board-eligible in nuclear medicine $(10.8 \%)$, radiology $(2.9 \%)$, or another specialty $(2.1 \%)$. Fifty-eight percent of medical directors were certified by the CBNC.

Nearly half $(45 \%)$ of technical directors held more than one registered credential relevant to nuclear imaging, with CNMT $(85.8 \%)$ and RT(N) $(44.8 \%)$ being the most prevalent. A minority $(8.9 \%)$ of technical directors additionally held either the NCT or PET credential, and 284 (14.8\%) held a parallel imaging credential such as RT(R), RT(CT), or RT (MR) in addition to the CNMT or RT(N) nuclear imaging credential.

Medical directors reported adequate CME relevant to nuclear cardiology in $74.8 \%$ of applications, and $74.5 \%$ of medical staff were compliant with the CME requirement. Technical directors reported adequate $\mathrm{CE}$ in $80.4 \%$ of applications, and $81.1 \%$ of technical staff were compliant with the $\mathrm{CE}$ requirement. For nearly half $(49.7 \%)$ of laboratories in which both the medical director and the technical director were compliant with the $\mathrm{CME} / \mathrm{CE}$ requirements, an initial decision was made to grant accreditation, compared with $24.5 \%$ of laboratories in which either the medical director or the technical director, or both, was not compliant with the $\mathrm{CME} / \mathrm{CE}$ requirements $(P<0.001$, Table 2).

TABLE 2

Characteristics of Nuclear Cardiology Laboratory Staffs (Physician and Technologist) Applying for IAC Nuclear/PET Accreditation, 2011-2014

\begin{tabular}{|c|c|c|c|c|}
\hline Characteristic & $\begin{array}{l}\text { All laboratories } \\
\quad(n=1,913)\end{array}$ & $\begin{array}{c}\text { Accreditation } \\
\text { granted }(n=779)\end{array}$ & $\begin{array}{c}\text { Accreditation } \\
\text { delayed }(n=1,134)\end{array}$ & $P$ \\
\hline \multicolumn{5}{|l|}{ Physician characteristics } \\
\hline Medical director years of experience (mean) & $17.47(\mathrm{SD}, 8.34)$ & $17.34(\mathrm{SD}, 8.36)$ & $17.55(\mathrm{SD}, 8.32)$ & 0.67 \\
\hline $\begin{array}{l}\text { Medical director annual number of } \\
\text { studies interpreted (mean) }\end{array}$ & $804.20(\mathrm{SD}, 1,273.8)$ & $746.46(\mathrm{SD}, 1,273.48)$ & $843.87(\mathrm{SD}, 1,273.07)$ & 0.06 \\
\hline Medical director with CBNC (n) & $1,107(57.9 \%)$ & $491(63.0 \%)$ & $616(54.3 \%)$ & $<0.001$ \\
\hline Medical director who is cardiologist $(n)$ & $1,330(69.5 \%)$ & $564(72.4 \%)$ & $766(67.5 \%)$ & 0.02 \\
\hline Medical director compliant with CME (n) & $1,432(74.9 \%)$ & $664(85.2 \%)$ & $768(67.7 \%)$ & $<0.001$ \\
\hline MPI volume per medical director (mean) & 475.79 (SD, 409.07$)$ & $468.94(\mathrm{SD}, 416.16)$ & 485.75 (SD, 398.57) & 0.92 \\
\hline Number of medical staff (mean) & $3.18(\mathrm{SD}, 5.04)$ & $3.39(\mathrm{SD}, 5.49)$ & $3.03(\mathrm{SD}, 4.71)$ & 0.05 \\
\hline Number of CBNC staff (mean) & 1.37 (SD, 2.50) & $1.58(\mathrm{SD}, 2.85)$ & $1.22(\mathrm{SD}, 2.22)$ & $<0.001$ \\
\hline Number of medical staff CME-compliant (mean) & $2.36(\mathrm{SD}, 4.10)$ & $2.83(\mathrm{SD}, 4.73)$ & $2.05(\mathrm{SD}, 3.58)$ & $<0.001$ \\
\hline \multicolumn{5}{|l|}{ Technologist characteristics } \\
\hline Technical director years of experience (mean) & $19.21(\mathrm{SD}, 10.12)$ & $19.95(\mathrm{SD}, 10.20)$ & $18.71(\mathrm{SD}, 10.03)$ & 0.35 \\
\hline Number of technologists (mean) & $2.55(\mathrm{SD}, 2.85)$ & $2.64(\mathrm{SD}, 2.83)$ & $2.49(\mathrm{SD}, 2.87)$ & 0.43 \\
\hline Number of technical staff (mean) & $1.55(\mathrm{SD}, 2.85)$ & $1.64(\mathrm{SD}, 2.84)$ & 1.49 (SD, 2.87) & 0.43 \\
\hline Technical director compliant with CME (n) & $1,538(80.4 \%)$ & $702(90.1 \%)$ & $836(73.7 \%)$ & $<0.001$ \\
\hline Number of technical staff CE-compliant (mean) & $1.26(\mathrm{SD}, 2.45)$ & $1.43(\mathrm{SD}, 2.57)$ & $1.14(\mathrm{SD}, 2.36)$ & 0.01 \\
\hline $\begin{array}{l}\text { Technical director with PET or NCT plus CNMT } \\
\text { or } \mathrm{RT}(\mathrm{N})(n)\end{array}$ & $166(8.7 \%)$ & $82(10.5 \%)$ & $84(7.40 \%)$ & 0.02 \\
\hline $\begin{array}{l}\text { Technical director with (RT[R], RT[CT], } \\
\text { or RT[MR]) plus CNMT or RT(N) }(n)\end{array}$ & $284(14.8 \%)$ & $117(15.0 \%)$ & $167(14.7 \%)$ & 0.86 \\
\hline
\end{tabular}


On multivariate adjustment, several variables were associated with increased odds that accreditation would be granted, including a medical director with CBNC certification, a technical director with NCT or PET credentials, medical and technical directors with $\mathrm{CME} / \mathrm{CE}$ compliance, many medical staff with CME/CE compliance, and the assistance of a consultant with the application (Table 3 ). The only variable that negatively affected the odds of receiving accreditation was the number of medical staff. The number of years of experience for either the medical or the technical director was not associated with the decision to grant or delay accreditation.

The exploratory analysis of the average annual number of studies interpreted per physician did not change the magnitude or direction of the associations in the primary analyses.

Variables that were independently associated with a decreased protocol deficiency were consultant assistance, medical director $\mathrm{CME}$ compliance, technical director $\mathrm{CE}$ compliance, and many medical staff with CME compliance. Variables associated with an increased protocol deficiency were laboratories that also performed general nuclear medicine or PET neurology/oncology studies, medical directors

TABLE 3

Impact of Laboratory Characteristics and Staff Training and Credentials on MPI Accreditation Decisions

\begin{tabular}{|c|c|}
\hline Characteristic & Adjusted odds ratio \\
\hline Number of sites & $1.01(0.97-1.05)$ \\
\hline $\begin{array}{l}\text { Annual laboratory volume per } \\
1,000 \mathrm{MPI} \text { studies }\end{array}$ & $1.06(0.96-1.18)$ \\
\hline $\begin{array}{l}\text { Application cycle (per subsequent } \\
\text { cycle) }\end{array}$ & $1.00(0.91-1.11)$ \\
\hline $\begin{array}{l}\text { Consultant assistance with } \\
\text { application }\end{array}$ & $1.94(1.49-2.53)$ \\
\hline Hospital facility & $1.08(0.75-1.57)$ \\
\hline $\begin{array}{l}\text { Doing general nuclear medicine } \\
\text { or PET oncology/neurology }\end{array}$ & $0.89(0.58-1.37)$ \\
\hline Medical director with CBNC status & $1.28(1.03-1.58)$ \\
\hline $\begin{array}{l}\text { Cardiovascular pathway of medical } \\
\text { director }\end{array}$ & $1.03(0.82-1.29)$ \\
\hline Medical director compliant with CME & $1.97(1.53-2.54)$ \\
\hline \multicolumn{2}{|l|}{$\begin{array}{l}\text { Volume of studies interpreted by } \\
\text { medical director, quartiles }\end{array}$} \\
\hline $0-300$ & Referent \\
\hline $301-500$ & $1.21(0.92-1.60)$ \\
\hline $501-900$ & $1.03(0.78-1.36)$ \\
\hline $901+$ & $0.75(0.55-1.03)$ \\
\hline Number of medical staff & $0.82(0.75-0.89)$ \\
\hline $\begin{array}{l}\text { At least } 50 \% \text { of medical staff } \\
\text { with CBNC }\end{array}$ & $1.06(0.85-1.31)$ \\
\hline $\begin{array}{l}\text { Number of medical staff compliant } \\
\text { with } \mathrm{CE}\end{array}$ & $1.28(1.16-1.42)$ \\
\hline $\begin{array}{l}\text { Technical director with NCT or } \\
\text { PET vs. CNMT or RT(N) }\end{array}$ & $1.49(1.06-2.10)$ \\
\hline Technical director compliant with CE & $2.48(1.86-3.31)$ \\
\hline Number of technical staff & $0.91(0.80-1.04)$ \\
\hline $\begin{array}{l}\text { Number of technical staff compliant } \\
\text { with CE }\end{array}$ & $1.12(0.97-1.29)$ \\
\hline
\end{tabular}

$95 \%$ confidence intervals are in parentheses. who had interpreted more than 900 studies, and a high number of medical staff (Table 4).

Variables that were independently associated with a decreased reporting deficiency were consultant assistance, a medical director with $\mathrm{CBNC}$ certification, medical director CME compliance, technical director $\mathrm{CE}$ compliance, and a technical director with NCT or PET credentials. The only variable that was associated with an increased reporting deficiency was the number of sites (Table 4).

\section{DISCUSSION}

This analysis of 1,913 nuclear cardiology laboratories demonstrated that most facilities seeking IAC Nuclear/PET accreditation for MPI are directed by CBNC-certified cardiologists and predominantly by registered technologists, many of whom hold multiple imaging credentials. Nuclear cardiology laboratories directed by CBNC-certified physicians and NCT- or PET-credentialed technologists were less likely to receive a decision to delay accreditation. Moreover, CBNC certification of medical directors was associated with increased odds of being granted accreditation and reduced odds of being found deficient in protocols and reporting, whereas the specialty and experience of the medical director were not. Together, these findings suggest that professionals with advanced training specific to nuclear cardiology are more likely to perform nuclear cardiology in the detail-oriented fashion that leads to granting of accreditation. Although these findings are not altogether surprising, they represent an important validation of the value of having specific training credentials in addition to general training in cardiology or radiation imaging technology. Additionally, directors and staff who complied with the IAC Nuclear/PET Standards for CME/CE were less likely to have deficiencies in protocols and reporting, an association suggesting that CME/CE activity can improve laboratory operations beyond the mere fulfillment of a statutory requirement for quantity of ongoing education.

In the overall accreditation decision, as well as in the domains of protocols and reporting, the impact of the training and continuing education of the technical director and technical staff is like that of the medical director and medical staff. This finding validates the common observation that technologists, responsible for patient preparation, image acquisition, instrument quality control, and data reporting (8), have importance equal to that of the interpreting physician in the operational quality of the imaging laboratory. The $\mathrm{CME} / \mathrm{CE}$ compliance of both the medical director and the technical director, but not the years of experience of either, was significantly associated with the granting of accreditation. This distinction emphasizes the importance of up-to-date knowledge on technologies and practice standards in the successful organization and operation of a nuclear cardiology laboratory, independent of duration of experience.

Facilities performing both cardiac and noncardiac imaging tended to have more deficiencies in cardiac imaging 
TABLE 4

Impact of Laboratory Characteristics and Staff Training on IAC Nuclear/PET Review Deficiencies in Protocols and Reporting

\begin{tabular}{|c|c|c|}
\hline Characteristic & $\begin{array}{l}\text { Adjusted odds ratio for protocol } \\
\text { deficiencies }\end{array}$ & $\begin{array}{c}\text { Adjusted odds ratio for reporting } \\
\text { deficiencies }\end{array}$ \\
\hline Number of sites & $1.06(0.96-1.19)$ & $1.08(1.02-1.13)$ \\
\hline Annual laboratory volume per $1,000 \mathrm{MPI}$ studies & $0.93(0.84-1.02)$ & $0.95(0.86-1.04)$ \\
\hline Application cycle (per subsequent cycle) & $1.07(0.96-1.19)$ & $1.00(0.91-1.10)$ \\
\hline Consultant assistance with application & $0.55(0.41-0.73)$ & $0.63(0.48-0.81)$ \\
\hline Hospital facility & $1.24(0.85-1.82)$ & $0.78(0.54-1.13)$ \\
\hline $\begin{array}{l}\text { Doing general nuclear medicine or PET } \\
\text { oncology/neurology }\end{array}$ & $1.70(1.12-2.56)$ & $0.99(0.66-1.49)$ \\
\hline Medical director with CBNC status & $0.88(0.71-1.09)$ & $0.78(0.63-0.95)$ \\
\hline Cardiovascular pathway of medical director & $0.99(0.79-1.24)$ & $0.82(0.66-1.02)$ \\
\hline Medical director compliant with CME & $0.55(0.44-0.69)$ & $0.64(0.51-0.80)$ \\
\hline \multicolumn{3}{|l|}{$\begin{array}{l}\text { Volume of studies interpreted by medical } \\
\text { director, quartiles }\end{array}$} \\
\hline $0-300$ & Referent & Referent \\
\hline $301-500$ & $0.87(0.65-1.16)$ & $0.91(0.70-1.20)$ \\
\hline $501-900$ & $0.98(0.74-1.30)$ & $0.93(0.71-1.22)$ \\
\hline $901+$ & $1.51(1.12-2.03)$ & $1.09(0.81-1.45)$ \\
\hline Number of medical staff & $1.16(1.08-1.24)$ & $1.04(0.97-1.11)$ \\
\hline At least $50 \%$ of medical staff with CBNC & $0.85(0.68-1.06)$ & $0.90(0.73-1.11)$ \\
\hline Number of medical staff compliant with CME & $0.84(0.77-0.91)$ & $0.94(0.87-1.02)$ \\
\hline $\begin{array}{l}\text { Technical director with NCT or PET vs. CNMT } \\
\text { or RT(N) }\end{array}$ & $0.79(0.55-1.13)$ & $0.67(0.47-0.95)$ \\
\hline Technical director compliant with CE & $0.56(0.44-0.72)$ & $0.53(0.41-0.67)$ \\
\hline Number of technical staff & $0.92(0.81-1.04)$ & $1.00(0.90-1.11)$ \\
\hline Number of technical staff compliant with CE & $1.06(0.92-1.21)$ & $0.98(0.87-1.11)$ \\
\hline
\end{tabular}

95\% confidence intervals are in parentheses.

protocols (MPI) than did facilities performing exclusively cardiac imaging. Applying for accreditation of many different types of protocols also increases the likelihood of errors and missing or outdated materials. Similarly, we hypothesize that the increased reporting deficiencies in facilities with multiple sites and large medical staffs are related both to the increased complexity of the application materials and to the increased possibility of variability across sites and among interpreting physicians.

Less clear is the finding of an association between a medical director whose volume of interpreted studies falls within the highest quartile and an increased number of protocol deficiencies. This may be a statistical false-negative finding, especially as greater volume has been associated with greater quality across a range of procedures, including coronary artery bypass grafting and acute myocardial infarction care (9). However, we are not aware of any studies relating the interpretative quality of nuclear imaging (i.e., diagnostic accuracy) to volume (10). One study of echocardiography interpretation demonstrated an increased frequency of errors when higher numbers of reports were signed per hour (11), but annual laboratory-volume data do not allow us to test whether speed is a factor in our sample.

The assistance of a consultant in preparing the application was strongly associated with the initial granting of accreditation as opposed to a delay, underscoring the way the accreditation process relies heavily on a snapshot of materials presented to the IAC and its reviewers (12).

A key limitation of this analysis is that accreditation is voluntary for hospital-based nuclear cardiology laboratories but mandatory for non-hospital-based facilities, resulting in a biased sample. This bias may be compounded by the fact that accreditation of imaging facilities is available not only through the IAC but also through the American College of Radiology and that laboratories seeking accreditation through one of these bodies may systematically differ from laboratories seeking accreditation through the other (13). Laboratory materials are reviewed for accreditation by a diverse group of physicians, technologists, and IAC Nuclear/PET specialists, although all reviewers undergo the same initial and periodically updated training. Our results may also have been biased by any changes in the leadership or staff of a laboratory during an accreditation cycle-changes that may occur independently of substantive changes in laboratory operations.

The possibility of personnel changes or laboratory mergers during an IAC Nuclear/PET accreditation period means that operations as described in the application materials may not reflect operations under the current leadership in some laboratories. These structural-quality data are distinct from outcomes such as diagnostic accuracy and improved patient care (14), and further studies will be required to explore the impact of advanced training credentials on patient outcomes. As with all observational research, we could adjust for only 
those variables that were included in the analysis. We cannot discount the possibility that other confounders may be influencing the results.

The American College of Cardiology core cardiovascular training statement was recently updated (15), and the new training standards had not been incorporated into the IAC Nuclear/PET Standards as of the time of this study.

\section{CONCLUSION}

In nuclear cardiology laboratories seeking IAC Nuclear/ PET accreditation, the presence of medical directors with CBNC certification and technical directors with specialized credentials is associated with increased odds of being granted accreditation and reduced odds of having deficiencies in protocols and reporting. These findings substantiate the association between specific training credentials, in addition to general training in cardiology or nuclear imaging technology, and improved-quality cardiovascular imaging. More research is required to link these structural quality measures with patient outcomes.

\section{DISCLOSURE}

This work was supported by an investigator-initiated research grant to the authors from the Intersocietal Accreditation Commission, Ellicott City, MD. The content is solely the responsibility of the authors and does not necessarily represent the official views of the IAC or the IAC Research Committee. No other potential conflict of interest relevant to this article was reported.

\section{ACKNOWLEDGMENT}

We acknowledge Mary Beth Farrell, CNMT, for assistance with data management.

\section{REFERENCES}

1. Cerqueira MD, Arrighi JA, Geiser EA. Physician certification in cardiovascular imaging: rationale, process, and benefits. JACC Cardiovasc Imaging. 2008;1:801808.

2. Heller GV, Katanick SL, Sloper T, Garcia M. Accreditation for cardiovascular imaging: setting quality standards for patient care. JACC Cardiovasc Imaging. 2008;1:390-397.

3. The IAC standards and guidelines for nuclear/PET accreditation. Intersocietal Accreditation Commission website. https://www.intersocietal.org/nuclear/standards/ IACNuclearPETStandards2016.pdf. Published 2016. Accessed December 18, 2017.

4. Wackers FJ, Bateman TM. Blueprint of the certification examination in nuclear cardiology. Certification Council of Nuclear Cardiology. J Nucl Cardiol. 1997;4:164168 .

5. Cerqueira MD, Berman DS, Di Carli MF, Schelbert HR, Wackers FJT, Williams KA. Task force 5: training in nuclear cardiology endorsed by the American Society of Nuclear Cardiology. J Am Coll Cardiol. 2008;51:368-374.

6. Certification exams. Nuclear Medicine Technology Certification Board website. https://www.nmtcb.org/certification-exams.php. Accessed December 18, 2017.

7. What is ARRT certification and registration? American Registry of Radiologic Technologists website. https://www.arrt.org/Certification. Accessed December 18, 2017.

8. Nuclear Medicine Technologist Scope of Practice and Performance Standards. Society of Nuclear Medicine and Molecular Imaging website. http://snmmi.files. cms-plus.com/NMT_Scope_of_Practice_Clinical_Performance_Standards_FINAL_62013.pdf. Approved June 2013. Accessed December 18, 2018

9. Halm EA, Lee C, Chassin MR. Interpreting the Volume-Outcome Relationship in the Context of Health Care Quality. Washington, DC: National Academies Press; 2000.

10. Eskandari M, Kramer CM, Hecht HS, Jaber WA, Marwick TH. Evidence base for quality control activities in cardiovascular imaging. JACC Cardiovasc Imaging. 2016;9:294-305.

11. Spencer KT, Arling B, Sevenster M, et al. Identifying errors and inconsistencies in real time while using facilitated echocardiographic reporting. J Am Soc Echocardiogr. 2015;28:88.e1-92.e1.

12. Douglas PS, Chen J, Gillam L, et al. Achieving quality in cardiovascular imaging II: proceedings from the Second American College of Cardiology-Duke University Medical Center Think Tank on Quality in Cardiovascular Imaging. JACC Cardiovasc Imaging. 2009;2:231-240.

13. Wackers FT. ICANL and ACR nuclear medicine accreditation: a comparison. J Nucl Med. 2000;41(5):26N-28N.

14. Donabedian A. Evaluating the quality of medical care. 2005;4:691-729.

15. Dilsizian V, Arrighi JA, Cohen RS, Miller TD, Solomon AJ, Udelson JE. COCATS 4 Task Force 6: training in nuclear cardiology. J Am Coll Cardiol. 2015;65:18001809. 